hep-th/9903240

\title{
ASYMPTOTIC SYMMETRY AND THE GENERAL BLACK HOLE SOLUTION IN $\mathrm{AdS}_{3}$ GRAVITY
}

\author{
YUHSUKE YOSHIDA and TAKAHIRO KUBOTA \\ Department of Physics \\ Graduate School of Science, Osaka University \\ Machikaneyama 1-1, Toyonaka \\ Osaka 560-0043, JAPAN
}

\begin{abstract}
We describe the Brown-Henneaux asymptotic symmetry of the general black holes in the Chern-Simons gauge theory of the gauge group $S L(2 ; \mathbf{R})_{L} \times$ $S L(2 ; \mathbf{R})_{R}$. We make it clear that the vector-like subgroup $S L(2 ; \mathbf{R})_{L+R}$ plays an essential role in describing the asymptotic symmetry consistently. We find a quite general black hole solution in the $A d S_{3}$ gravity theory. The solution is specified by an infinite number of conserved quantities which constitute a family of mapping from $S^{1}$ to the gauge group. The BTZ black hole is one of the simplest case.
\end{abstract}

PACS number: 04.70.Bw, 04.20.Jb, 04.60.Kz, 11.25.Hf 


\section{Introduction}

There has been recently a surge of renewed interest in gravity and supergravity in anti-de Sitter $(A d S)$ space. This was kindled by remarkable observations [1, 2, 3, 4, 5, 6, 7, 8] that $A d S$ gravity in $(d+1)$-dimensions would describe the $d$-dimensional conformal field theory on its boundary. In the case of $A d S_{3}$ gravity, the occurrence of the two-dimensional conformal symmetry was elucidated by Brown and Henneaux 90 more than a decade ago. Their analysis, which is based on the work of Regge and Teitelboim[10], has been investigated by using the Chern-Simons gauge theory [11, 12] to a considerable extent 13, 14, 15, 16]. The salient feature of the Chern-Simons approach is that, although the gauge fields do not have local excitations, the gauge degrees of freedom on the boundary have non-trivial physical contents.

In the present paper we reanalyze the $A d S_{3}$ gravity. We will work with the ChernSimons gauge theory with the gauge group $G=S O(2,2)=S L(2 ; \mathbf{R})_{L} \times S L(2 ; \mathbf{R})_{R}$ throughout. We will pay a particular attention to the properties of diffeomorphism together with the gauge symmetry. To study the asymptotic symmetry of Brown and Henneaux one has to look for the diffeomorphism that leaves the metric $g_{m n}$ unchanged. In the dreibein formalism, on the other hand, the gauge transformation should also be taken into account in addition to the diffeomorphism to derive the asymptotic symmetry. As we will see, this is because the metric $g_{m n}$ is invariant under the gauge transformation in the vector-like subgroup $H=S L(2 ; \mathbf{R})_{L+R} \subset G$, while the dreibein $e_{m}^{a}$ is not. This means that we are necessarily led to consider a combination of diffeomorphism $\left(\delta_{D}\right)$ and the gauge transformation $\left(\delta_{H}\right)$ in $H$. The isometry condition is now

$$
\left(\delta_{D}+\delta_{H}\right) e_{m}^{a}=0
$$

We will show that the asymptotic symmetry is obtained by solving eq. (1.1) on the boundary at infinity. The solution of the isometry condition (1.1) at infinity contains infinite degrees of freedom and the asymptotic symmetry is thus infinite dimensional. A subtle feature of our analysis in contrast to previous works is that we consider transformations $\delta_{D}$ and $\delta_{H}$ simultaneously, while a full use of the subgroup $H$ and $\delta_{H}$ eluded previous authors.

It is expected that the generators of the above asymptotic symmetry are materialized by the Kac-Moody currents. There has been a tacit understanding in $A d S_{3}$ gravity theory that the representation space of the asymptotic symmetry bears close connections with 
the black hole and vacuum solutions. We will in fact uncover a quite general class of black hole solutions, to which the celebrated BTZ black hole 17 and vacuum solutions belong as the simplest cases. As will be seen, the black hole solutions are characterized by mappings from $S^{1}$ onto the gauge group $G$; the Kac-Moody currents.

The present paper is organized as follows. In sect. 2, the Chern-Simons formulation to the three dimensional gravity is briefly summarized. The boundary conditions for the gauge fields and the surface terms in the action are specified. The asymptotic symmetry on the boundary are derived by solving eq. (1.1) in sect. 3. The Kac-Moody and Virasoro algebras are worked out in sect. 1 and the classical part of the Virasoro central charge is derived in agreement with the previous analyses [9]. It is shown that the Brown-Henneaux asymptotic symmetry is associated not with the usual Sugawara form but with the twisted Sugawara form. In sect. 5 general black hole solutions are derived quite generally. Sect. 6 is devoted to the brief discussion of the quantum calculation of the Virasoro central charge. We will summarize our results in sect. 7 .

\section{$2 \mathrm{AdS}_{3}$ gravity and Chern-Simons theory}

In this paper we consider the three-dimensional gravity with a negative cosmological constant. The Einstein-Hilbert action is

$$
I_{E H}\left(g_{m n}\right)=\frac{1}{16 \pi G_{N}} \int d^{3} x \sqrt{-g}\left(R+\frac{2}{\ell^{2}}\right)
$$

This system has the BTZ black hole solution[17]. This system also has the asymptotic symmetry, which was first derived in ref. [9]. One of our main purpose is to derive the asymptotic symmetry in the BTZ black hole background using the Chern-Simons formulation with a closer look at the role of the subgroup $H$.

In this section we review the three-dimensional gravity described by eq. (2.1). Let us rewrite the action (2.1) in the dreibein formulation

$$
I_{E H}(e, \omega)=-\frac{1}{8 \pi G_{N}} \int\left(e_{a} R^{a}+\frac{1}{6 \ell^{2}} \epsilon_{a b c} e^{a} e^{b} e^{c}\right)+\frac{1}{16 \pi G_{N}} \oint e_{a} \omega^{a} .
$$

To avoid confusion we fix our notation now. We define the three-dimensional coordinate as $(t, \phi, \rho)$, the complete antisymmetric tensors as $\epsilon^{t \phi \rho}=\epsilon^{012}=+1$ and the flat metric and the local Lorentz frame metric as $\eta_{m n}=\eta_{a b}=\operatorname{diag}(-,+,+)$. We consider the system in

the cylinder $\mathbf{R} \times \Sigma$ where $\mathbf{R}$ is parameterized by the time $t$, the disc $\Sigma$ is parameterized by 
the angle and radial variables $(\phi, \rho)$ and, especially, the boundary $\mathbf{R} \times \partial \Sigma$ is parameterized by $(t, \phi)$.

The first term of the action (2.2) is rewritten as the Chern-Simons action with the gauge group $G=S O(2,2)=S L(2 ; \mathbf{R})_{L} \times S L(2 ; \mathbf{R})_{R}$. 112 Both $S L(2 ; \mathbf{R})_{L}$ and $S L(2 ; \mathbf{R})_{R}$ generators are represented by

$$
J_{0}=\frac{1}{2}\left(\begin{array}{cc}
0 & -1 \\
1 & 0
\end{array}\right), \quad J_{1}=\frac{1}{2}\left(\begin{array}{ll}
0 & 1 \\
1 & 0
\end{array}\right), \quad J_{2}=\frac{1}{2}\left(\begin{array}{cc}
1 & 0 \\
0 & -1
\end{array}\right) .
$$

Then, we have $\left[J_{a}, J_{b}\right]=\epsilon_{a b}^{c} J_{c}, \operatorname{tr} J_{a} J_{b}=\eta_{a b} / 2$. The combinations $A_{m}^{a} \equiv \omega_{m}^{a}+e_{m}^{a} / \ell$ and $B_{m}^{a} \equiv \omega_{m}^{a}-e_{m}^{a} / \ell$ become the $S L(2 ; \mathbf{R})_{L}$ and $S L(2 ; \mathbf{R})_{R}$ gauge fields, respectively. The Chern-Simons action is

$$
I_{C S}(A)=\frac{k}{4 \pi} \operatorname{tr} \int\left(A d A+\frac{2}{3} A^{3}\right) .
$$

Then, the first term of the action (2.2) becomes $I_{C S}(B)-I_{C S}(A)$ with $k=\ell /\left(4 G_{N}\right)$. In what follows, all the quantities are regarded as dimensionless being rescaled by the parameter $\ell$.

Let us consider the boundary condition of the gauge fields. What we would like to adopt is the boundary condition 18, 19

$$
A_{t}=A_{\phi}, \quad B_{t}=-B_{\phi},
$$

at asymptotic infinity $\rho \sim \infty$. This is the only appropriate boundary condition as will be seen later. The variation of the Chern-Simons action is

$$
\delta I_{C S}(B)-\delta I_{C S}(A)=\frac{k}{2 \pi} \operatorname{tr} \int\left(\delta B F_{B}-\delta A F_{A}\right)+\delta \mathcal{B}^{\prime},
$$

where

$$
\delta \mathcal{B}^{\prime}=\frac{k}{4 \pi} \operatorname{tr} \oint\left(\delta B_{t} B_{\phi}-\delta B_{\phi} B_{t}-\delta A_{t} A_{\phi}+\delta A_{\phi} A_{t}\right) .
$$

What is needed is the boundary term $\mathcal{B}$ to be added to the action $I_{C S}(B)-I_{C S}(A)$ such that the condition $\delta \mathcal{B}+\delta \mathcal{B}^{\prime}=0$ provides us with the boundary condition (2.5). The desirable boundary term turns out to be 20

$$
\mathcal{B}=\frac{k}{8 \pi} \operatorname{tr} \oint\left(A_{t}^{2}-A_{\phi}^{2}+B_{t}^{2}-B_{\phi}^{2}\right) .
$$

We notice that the functional derivatives $\delta / \delta A$ and $\delta / \delta B$ become well-defined in this case. In accordance with the boundary condition (2.5), the gauge functions $u \in \operatorname{sl}(2 ; \mathbf{R})_{L}$ and $v \in \operatorname{sl}(2 ; \mathbf{R})_{R}$ also have the boundary condition $\partial_{t} u=\partial_{\phi} u$ and $\partial_{t} v=-\partial_{\phi} v$. Adding the boundary term $\mathcal{B}$, the desirable action is

$$
L=I_{C S}(B)-I_{C S}(A)+\mathcal{B} .
$$


Now, we postulate that the action of the system be $L$. We will justify the boundary term in sect. 5 .

Let us find a solution of the action (2.9). The more general solution will be found in sect. 5. The Einstein equation and torsion free condition are equivalently expressed by the equations of motion $F_{A} \equiv d A+A^{2}=0$ and $F_{B} \equiv d B+B^{2}=0$. In this section we would like to find the BTZ black hole solution. We will first find a constant solution $d A=d B=0$, and show that this is gauge-transformed to the BTZ solution. We tentatively use the gauge fixing condition $A_{\rho}=B_{\rho}=0$ only for the purpose of finding a constant solution. Now, the equations of motion are $\left[A_{t}, A_{\phi}\right]=\left[B_{t}, B_{\phi}\right]=0$. These can be solved by setting $A_{\phi}=A_{t}$ and $B_{\phi}=-B_{t}$. These relations are required from the boundary condition (2.5). The quantities $A_{t}$ and $B_{t}$ may be any elements of the Lie algebra $\operatorname{sl}(2 ; \mathbf{R})$. Each choice of the elements singles out various (black hole) solutions. For example, the case $A_{t}=-B_{t}=J_{0}$ corresponds to the anti-de-Sitter space $d s^{2}=-\left(r^{2}+1\right) d t^{2}+\left(r^{2}+1\right)^{-1} d r^{2}+r^{2} d \phi^{2}$ as will be seen in sect. 5. For deriving the BTZ solution, we take $A_{t}=z_{+} J_{1}$ and $B_{t}=z_{-} J_{1}$ by introducing two arbitrary constants $z_{ \pm}$. Then, we obtain

$$
\begin{aligned}
A & =\frac{z_{+}}{2}\left(\begin{array}{cc}
0 & d t+d \phi \\
d t+d \phi & 0
\end{array}\right), \\
B & =\frac{z_{-}}{2}\left(\begin{array}{cc}
0 & d t-d \phi \\
d t-d \phi & 0
\end{array}\right) .
\end{aligned}
$$

Next, we perform the gauge transformation $g=\exp \rho J_{2}$ as $A \rightarrow g^{-1} A g+g^{-1} d g$ and $B \rightarrow g B g^{-1}+g d g^{-1}$, which is belonging to $G / H$. We have

$$
\begin{aligned}
A & =\frac{1}{2}\left(\begin{array}{cc}
d \rho & z_{+} e^{-\rho}(d t+d \phi) \\
z_{+} e^{\rho}(d t+d \phi) & -d \rho
\end{array}\right) \\
& =z_{+}\left(J_{0} \sinh \rho+J_{1} \cosh \rho\right)(d t+d \phi)+J_{2} d \rho \\
B & =\frac{1}{2}\left(\begin{array}{cc}
-d \rho & z_{-} e^{\rho}(d t-d \phi) \\
z_{-} e^{-\rho}(d t-d \phi) & d \rho
\end{array}\right) \\
& =z_{-}\left(J_{0} \sinh \rho-J_{1} \cosh \rho\right)(d t-d \phi)-J_{2} d \rho .
\end{aligned}
$$

The tentative gauge fixing condition changes to $A_{\rho}=J_{2}$ and $B_{\rho}=-J_{2}$. It is interesting that radial functional form is introduced as the broken gauge degrees of freedom. Here we make a comment on $G / H$. Unless we perform the gauge transformation $g \in G / H$, the solution (2.10) would produce an unphysical degenerate metric. On the other hand, the metric determined by the solution (2.11) is not degenerate, as is seen below, and has physical significance. Namely, the gauge transformation $G / H$ connecting between the two 
solutions has a physical meaning. Expanding the above in terms of the generators (2.3) and using the relations $A=\omega+e$ and $B=\omega-e$, we finally obtain

$$
\begin{array}{ll}
e^{0}=\sinh \rho\left(r_{+} d t-r_{-} d \phi\right), & \omega^{0}=\sinh \rho\left(r_{+} d \phi-r_{-} d t\right), \\
e^{1}=\cosh \rho\left(r_{+} d \phi-r_{-} d t\right), & \omega^{1}=\cosh \rho\left(r_{+} d t-r_{-} d \phi\right), \\
e^{2}=d \rho, & \omega^{2}=0,
\end{array}
$$

where we put $z_{ \pm}= \pm r_{+}-r_{-}$. Reparameterizing the radial coordinate as $r^{2}=r_{+}^{2} \cosh ^{2} \rho-$ $r_{-}^{2} \sinh ^{2} \rho$ allows us to obtain the standard form of the BTZ black hole geometry [17]

$$
\begin{aligned}
d s^{2}= & \eta_{a b} e^{a} e^{b} \\
= & -N^{2} d t^{2}+N^{-2} d r^{2}+r^{2}\left(d \phi+N_{\phi} d t\right)^{2}, \\
& N^{2}=\frac{\left(r^{2}-r_{+}^{2}\right)\left(r^{2}-r_{-}^{2}\right)}{r^{2}} \\
& N_{\phi}=-\frac{r_{+} r_{-}}{r^{2}} .
\end{aligned}
$$

If we regard $r_{+}$and $r_{-}$as the outer and inner horizons, respectively, then we have $r_{+} \geq$ $r_{-}>0$. We notice that $\rho=0$ corresponds to the event horizon $r=r_{+}$.

The solution can be extended to inside of the event horizon. 21 However, since we are interested in the asymptotic behavior of the system at infinity, we will concentrate on the outside of the event horizon.

\section{$3 \quad$ Asymptotic symmetry at infinity}

In the Chern-Simons theory the diffeomorphism of the gauge field is embedded into the gauge transformation. The embedded diffeomorphism becomes an on-shell symmetry. Since the background satisfies the on-shell condition, the embedded diffeomorphism makes sense when we consider the Brown-Henneaux asymptotic symmetry. In the present system, under consideration, the diffeomorphisms of the gauge fields $A$ and $B$ are embedded into the gauge group $S L(2 ; \mathbf{R})_{L}$ and $S L(2 ; \mathbf{R})_{R}$, respectively. What we will show is that the Brown-Henneaux asymptotic symmetry is not merely this usual diffeomorphism but a superposition of the usual diffeomorphism and a gauge transformation. We would like to emphasize that the isometry is given by

$$
u^{a}=\xi^{m} A_{m}^{a}+w^{a}, \quad v^{a}=\xi^{m} B_{m}^{a}+w^{a},
$$

where $w^{a}$ is a gauge function depending on $\xi^{m}$. This means that the isometry is not the usual diffeomorphism in the Chern-Simons formulation. The gauge functions (3.1) are 
in a sharp contrast with the prescription used in refs. [13, 19]. Unless we consider the gauge function $w^{a}$ in (3.1), one would be forced to introduce two kinds of diffeomorphism parameter $\xi^{m}$ in $u^{a}$ and $v^{a}$ separately. This is hardly acceptable. It should be stressed that our result (3.1) is the only consistent way to explain the Brown-Henneaux asymptotic symmetry in the Chern-Simons formulation. In the following we derive the form (3.1) in more detail.

The diffeomorphism, with parameter $\xi^{m}$, of one-form is given by $\delta_{D} A_{m}=\xi^{n} \partial_{n} A_{m}+$ $\partial_{m} \xi^{n} A_{n}$. At on-shell $F_{m n}=0$ this can be embedded into the gauge transformation. The infinitesimal gauge transformations of the left- and right-handed sectors are given by $\delta_{L} A=d u+[A, u]$ and $\delta_{R} B=d v+[B, v]$, so the gauge transformations for the dreibein and spin connection are

$$
\begin{aligned}
& \delta_{G} e_{m}^{a}=\frac{1}{2}\left(\delta_{L} A_{m}^{a}-\delta_{R} B_{m}^{a}\right)=\partial_{m} u_{-}^{a}+\epsilon_{b c}^{a}\left(\omega_{m}^{b} u_{-}^{c}+e_{m}^{b} u_{+}^{c}\right), \\
& \delta_{G} \omega_{m}^{a}=\frac{1}{2}\left(\delta_{L} A_{m}^{a}+\delta_{R} B_{m}^{a}\right)=\partial_{m} u_{+}^{a}+\epsilon_{b c}^{a}\left(\omega_{m}^{b} u_{+}^{c}+e_{m}^{b} u_{-}^{c}\right),
\end{aligned}
$$

where $u_{ \pm}^{a}=\left(u^{a} \pm v^{a}\right) / 2$. Note that we are using the quantities $u^{a}$ and $v^{a}$ as generic $S L(2 ; \mathbf{R})_{L}$ and $S L(2 ; \mathbf{R})_{R}$ gauge functions, respectively. The on-shell diffeomorphisms of the gauge fields are expressed by $u^{a}=\xi^{m} A_{m}^{a}$ and $v^{a}=\xi^{m} B_{m}^{a}$, respectively. These gauge functions $u^{a}$ and $v^{a}$ define the embedded diffeomorphism of the dreibein $\delta_{D} e_{m}^{a}$ and the spin connection $\delta_{D} \omega_{m}^{a}$ through eqs. (3.2). The diffeomorphism of the dreibein $\delta_{D} e_{m}^{a}$ induces that of the metric $g_{m n}=\eta_{a b} e_{m}^{a} e_{n}^{b}$;

$$
\delta_{D} g_{m n}=\xi^{l} \partial_{l} g_{m n}+\partial_{m} \xi^{l} g_{l n}+\partial_{n} \xi^{l} g_{m l}
$$

So, one might think that the isometry group defined by $\delta_{D} g_{m n}=0$ would be found by the condition $\delta_{D} e_{m}^{a}=0$. However, this is not correct. We have to take account of the gauge degrees of freedom in $H$.

The gauge transformation of the dreibein (3.2) induces that of the metric

$$
\delta_{G} g_{m n}=\eta_{a b}\left(e_{m}^{a} D_{n} u_{-}^{b}+e_{n}^{a} D_{m} u_{-}^{b}\right)
$$

where $D_{m} u_{-}^{a} \equiv \partial_{m} u_{-}^{a}+\epsilon_{b c}^{a} \omega_{m}^{b} u_{-}^{c}$. Since the metric is not a gauge invariant quantity of the gauge group $G$, the presence of the metric triggers the spontaneous symmetry breaking of the gauge symmetry $G$. The presence of the metric, namely in a broken phase, does not mean the breaking of the whole gauge symmetry $G$, but preserves a part of the symmetry $H \subset G$. The invariance of the metric $\delta_{G} g_{m n}=0$ means

$$
u=v,
$$


which defines the manifest symmetry $H$ in the broken phase. Namely, the metric is still invariant under the gauge transformation

$$
\delta_{H} e_{m}^{a}=\epsilon_{b c}^{a} e_{m}^{b} w^{c}
$$

where we put $u_{-}=0$ and $u_{+}=w$ in the transformation (3.2). This is an $S O(2,1)$ rotation, with parameter $w$, in the local frame. The manifest symmetry $H$ is recognized as the vector-like subgroup $S L(2 ; \mathbf{R})_{L+R}$ defined by eq. (3.5).

In conclusion, the condition of the isometry $\delta_{D} g_{m n}=0$ should be interpreted, in the dreibein formulation, as the condition

$$
\delta_{D} e_{m}^{a}+\delta_{H} e_{m}^{a}=\xi^{n} \partial_{n} e_{m}^{a}+\partial_{m} \xi^{n} e_{n}^{a}+\epsilon_{b c}^{a} e_{m}^{b} w^{c}=0
$$

Of course, if we did not use the dreibein, the condition $\delta_{D} g_{m n}=0$ determines $\xi^{m}$ consistently. However, the gauge degrees of freedom $w^{a}$ could not be determined. The condition (3.7) determines both of the diffeomorphism function $\xi^{m}$ and associating gauge function $w^{a}$ in eqs. (3.1).

Next, let us solve the isometry condition (3.7). By using the concrete form of the dreibein (2.12), the isometry condition becomes

$$
\begin{gathered}
\frac{\hat{g}_{j k}}{\left(r_{+}^{2}-r_{-}^{2}\right) \cosh \rho \sinh \rho} \partial_{i} \xi^{k}+\xi^{\rho} \eta_{i j}-w^{2} \epsilon_{i j}=0 \\
w^{a}=-\epsilon_{b}^{a} e_{i}^{b} \partial_{\rho} \xi^{i} \\
\partial_{i} \xi^{\rho}=-\hat{g}_{i j} \partial_{\rho} \xi^{j}
\end{gathered}
$$

where $a, b=0,1, \epsilon^{a b} \equiv \epsilon^{a b 2}, i, j=t, \phi, \xi_{i} \equiv \eta_{i j} \xi^{j}$ and

$$
\hat{g}_{i j}=\left(\begin{array}{cc}
r_{-}^{2} \cosh ^{2} \rho-r_{+}^{2} \sinh ^{2} \rho & -r_{+} r_{-} \\
-r_{+} r_{-} & r_{+}^{2} \cosh ^{2} \rho-r_{-}^{2} \sinh ^{2} \rho
\end{array}\right) .
$$

Let us investigate the isometry condition (3.8) at infinity $\rho \sim \infty$. In the leading order in $e^{\rho}$ it becomes

$$
\begin{gathered}
\partial_{i} \xi_{j}+\xi^{\rho} \eta_{i j}-w^{2} \epsilon_{i j}=0 \\
\partial_{\rho}\left(\xi^{t} \pm \xi^{\phi}\right)=\frac{8 e^{-2 \rho}}{r_{+}^{2}-r_{-}^{2}} \partial_{\mp} \xi^{\rho} \\
w^{0} \pm w^{1}=\frac{ \pm r_{+}-r_{-}}{2} e^{\rho} \partial_{\rho}\left(\xi^{t} \pm \xi^{\phi}\right) .
\end{gathered}
$$

Especially, the symmetric part of the first condition is

$$
\partial_{i} \xi_{j}+\partial_{j} \xi_{i}+2 \xi^{\rho} \eta_{i j}=0
$$


This is the condition for the conformal Killing vector in the asymptotic region at infinity. We should note that if we did not include the gauge symmetry contribution (3.6), say $w=0$, we would not obtain the conformal symmetry. The solution of the condition (3.10) is

$$
\begin{aligned}
\xi^{t} \pm \xi^{\phi} & =2 T_{ \pm}+\frac{2 e^{-2 \rho}}{r_{+}^{2}-r_{-}^{2}} \partial_{\mp}^{2} T_{\mp}, \\
\xi^{\rho} & =-\left(\partial_{+} T_{+}+\partial_{-} T_{-}\right), \\
w^{0} \pm w^{1} & =-\frac{4 e^{-\rho}}{ \pm r_{+}+r_{-}} \partial_{\mp}^{2} T_{\mp}, \\
w^{2} & =-\partial_{+} T_{+}+\partial_{-} T_{-},
\end{aligned}
$$

where $T_{ \pm}=T_{ \pm}(t \pm \phi)$ are arbitrary function.

We may also hope to investigate the isometry condition (3.8) near horizon $\rho \sim 0$. However, the first condition of eqs. (3.8) diverges, and we should change the coordinates to regular ones like Kruskal coordinates. The condition (3.8) is not so easily solved in general $\rho$, but on any slice surface with constant $\rho$ the isometry is determined by the condition (3.8). As will be shown in sect. 5, this is manifestly seen by the fact that the boundary theory of the Chern-Simons theory is described by the Wess-Zumino-Witten action.

\section{Current algebras of Chern-Simons theory}

Following refs. [9, 10, 13] we define the first class constraint in the presence of the boundary. In this section we derive the Kac-Moody currents and the Virasoro generators making good use of the asymptotic symmetry (3.1).

The Chern-Simons action (2.9) defines the Poisson's brackets

$$
\begin{aligned}
\left\{A_{i}^{a}(x), A_{j}^{b}(y)\right\}_{P} & =-\frac{4 \pi}{k} \epsilon_{i j} \eta^{a b} \delta^{2}(x-y), \\
\left\{B_{i}^{a}(x), B_{j}^{b}(y)\right\}_{P} & =\frac{4 \pi}{k} \epsilon_{i j} \eta^{a b} \delta^{2}(x-y), \\
\left\{A_{i}^{a}(x), B_{j}^{b}(y)\right\}_{P} & =0
\end{aligned}
$$

where $i, j=\phi, \rho$ and $x, y \in \mathbf{R} \times \partial \Sigma$. The first class constraint is $F_{A i j} \approx F_{B i j} \approx 0$, and their integrated forms are

$$
g_{A}(\Lambda)=\frac{k}{4 \pi} \operatorname{tr} \int_{\Sigma} \epsilon^{i j} F_{A i j} \Lambda
$$

and similarly for $g_{B}(\Lambda)$. For later convenience the gauge function $\Lambda$ does or does not depend on the gauge fields. In order to calculate the Poisson's bracket of the first class 
constraint, they should be varied properly even on the boundary. Then, it is necessary to add a boundary term $Q_{A}(\Lambda)$ as $g_{A}(\Lambda) \rightarrow G_{A}(\Lambda) \equiv g_{A}(\Lambda)+Q_{A}(\Lambda)$ and similarly for $g_{B}(\Lambda)$. 113 The boundary terms are defined, in the variational form, by

$$
\begin{aligned}
& \delta Q_{A}(\Lambda)=-\frac{k}{2 \pi} \operatorname{tr} \int_{\Sigma} \epsilon^{i j} \partial_{i}\left(\delta A_{j} \Lambda\right), \\
& \delta Q_{B}(\Lambda)=-\frac{k}{2 \pi} \operatorname{tr} \int_{\Sigma} \epsilon^{i j} \partial_{i}\left(\delta B_{j} \Lambda\right) .
\end{aligned}
$$

Then, weakly we have

$$
\begin{aligned}
& \left\{A_{i}^{a}(x), G_{A}(\Lambda)\right\}_{P} \approx \partial_{i} \Lambda^{a}+\epsilon^{a}{ }_{b c} A_{i}^{b} \Lambda^{c}, \\
& \left\{B_{i}^{a}(x), G_{B}(\Lambda)\right\}_{P} \approx-\partial_{i} \Lambda^{a}-\epsilon_{b c}^{a} B_{i}^{b} \Lambda^{c} .
\end{aligned}
$$

Since the boundary term $Q_{A}$ is weakly equivalent to $G_{A}$, we may define the Poisson's bracket between the boundary terms as

$$
\left.\left\{Q_{A}(\alpha), Q_{A}(\beta)\right\}_{P} \equiv\left\{G_{A}(\alpha), G_{A}(\beta)\right\}_{P}\right|_{g_{A} \approx 0}
$$

and similarly for $Q_{B}$.

First, let us derive the generators of the gauge transformation and their Poisson's bracket relations; namely the Kac-Moody algebra. If the gauge function $\Lambda$ does not depend on the gauge field, this is immediately integrated to be

$$
\begin{aligned}
Q_{A}(\Lambda) & =-\frac{k}{2 \pi} \operatorname{tr} \int_{\Sigma} \epsilon^{i j} \partial_{i}\left(A_{j} \Lambda\right)=\frac{k}{2 \pi} \int_{0}^{2 \pi} d \phi \operatorname{tr}\left(A_{\phi} \Lambda\right), \\
Q_{B}(\Lambda) & =\frac{k}{2 \pi} \int_{0}^{2 \pi} d \phi \operatorname{tr}\left(B_{\phi} \Lambda\right),
\end{aligned}
$$

where we suppressed the integration constant. Notice that $\epsilon^{\rho \phi}=-1$. Therefore, we obtain

$$
\begin{aligned}
& \left\{Q_{A}(\alpha), Q_{A}(\beta)\right\}_{P}=-Q_{A}([\alpha, \beta])-\frac{k}{2 \pi} \operatorname{tr} \int_{\Sigma} \epsilon^{i j} \partial_{i}\left(\alpha \partial_{j} \beta\right), \\
& \left\{Q_{B}(\alpha), Q_{B}(\beta)\right\}_{P}=Q_{B}([\alpha, \beta])+\frac{k}{2 \pi} \operatorname{tr} \int_{\Sigma} \epsilon^{i j} \partial_{i}\left(\alpha \partial_{j} \beta\right) .
\end{aligned}
$$

Let us expand the gauge fields in terms of the Fourier modes $J_{n}^{a}$ and $\bar{J}_{n}^{a}$ as

$$
\begin{aligned}
A_{\phi}^{a} & =-\frac{2}{k} \sum_{n} J_{n}^{a} e^{-i n(t+\phi)}, \\
B_{\phi}^{a} & =\frac{2}{k} \sum_{n} \bar{J}_{n}^{a} e^{-i n(t-\phi)} .
\end{aligned}
$$


Here, the overall coefficients in eqs. (4.8) are determined such that the resulting KacMoody algebras take the standard form. The reality condition of the gauge fields $\left(A_{m}^{a}\right)^{\dagger}=$ $A_{m}^{a}$ and $\left(B_{m}^{a}\right)^{\dagger}=B_{m}^{a}$ implies $\left(J_{n}^{a}\right)^{\dagger}=J_{-n}^{a}$ and $\left(\bar{J}_{n}^{a}\right)^{\dagger}=\bar{J}_{-n}^{a}$. The Kac-Moody algebra reads

$$
\begin{aligned}
i\left\{J_{n}^{a}, J_{m}^{b}\right\}_{P} & =i \epsilon_{c}^{a b} J_{n+m}^{c}+\frac{k}{2} n \eta^{a b} \delta_{n+m}, \\
i\left\{\bar{J}_{n}^{a}, \bar{J}_{m}^{b}\right\}_{P} & =i \epsilon_{c}^{a b} \bar{J}_{n+m}^{c}+\frac{k}{2} n \eta^{a b} \delta_{n+m} .
\end{aligned}
$$

Here we make a comment on the definition of the currents $J_{n}^{a}$ and $\bar{J}_{n}^{a}$. The $\rho$ dependence of the gauge field components (4.8) arises through the homogeneous gauge transformation belonging to $G / H$. Strictly speaking, this dependence should be eliminated from the currents by performing an inverse homogeneous gauge transformation. For details, the reader is referred to eqs. (5.1) and (5.4).

Next, let us derive the generators of the isometry embedded into the gauge symmetry and their Poisson's bracket relations; namely the Virasoro algebra. The gauge fields satisfy the boundary condtion (2.5) and the gauge fixing condition $A_{\rho}=J_{2}$ and $B_{\rho}=-J_{2}$. So, the twisted diffeomorphism (3.1) with (3.12) becomes

$$
\begin{aligned}
& u=2 T_{+} A_{\phi}-2 \partial_{+} T_{+} \alpha \\
& v=-2 T_{-} B_{\phi}+2 \partial_{-} T_{-} \alpha
\end{aligned}
$$

where we define $\alpha=J_{2}$. It is a subtle fact that these quantities $u$ and $v$ satisfy the boundary condition, although the components $\xi^{\rho}$ and $w^{2}$ in the solution (3.12) do not. Then, after the integration of eq. (4.3), we obtain

$$
\begin{aligned}
Q_{A}(\xi) & =\frac{k}{2 \pi} \int_{0}^{2 \pi} d \phi T_{+} \operatorname{tr}\left(A_{\phi}^{2}+2 \alpha \partial_{+} A_{\phi}\right) \\
Q_{B}(\xi) & =-\frac{k}{2 \pi} \int_{0}^{2 \pi} d \phi T_{-} \operatorname{tr}\left(B_{\phi}^{2}+2 \alpha \partial_{-} B_{\phi}\right) .
\end{aligned}
$$

On putting $T_{ \pm}=e^{i n(t \pm \phi)}$, these boundary terms define the Virasoro generators

$$
\begin{aligned}
& L_{n}=\frac{1}{2} Q_{A}(\xi)=\frac{1}{k} \sum_{m} J_{a m} J_{n-m}^{a}+i n \alpha_{a} J_{n}^{a}+a_{0} \delta_{n}, \\
& \bar{L}_{n}=-\frac{1}{2} Q_{B}(\xi)=\frac{1}{k} \sum_{m} \bar{J}_{a m} \bar{J}_{n-m}^{a}-i n \alpha_{a} \bar{J}_{n}^{a}+a_{0} \delta_{n},
\end{aligned}
$$

where we added a constant $a_{0}=k / 4$. We see that the Virasoro generators are not the usual energy-momentum tensor, i.e., not the Sugawara form, but the twisted energy-momentum tensor

$$
\begin{aligned}
& T \rightarrow T+\partial_{+} J^{2} \\
& \bar{T} \rightarrow \bar{T}+\partial_{-} \bar{J}^{2} .
\end{aligned}
$$


Using eqs. (4.9), we arrive at the Poisson's bracket relations

$$
\begin{aligned}
i\left\{L_{n}, L_{m}\right\}_{P} & =(n-m) L_{n+m}+\frac{k}{2} n\left(n^{2}-1\right) \delta_{n+m}, \\
i\left\{\bar{L}_{n}, \bar{L}_{m}\right\}_{P} & =(n-m) \bar{L}_{n+m}+\frac{k}{2} n\left(n^{2}-1\right) \delta_{n+m} .
\end{aligned}
$$

Now, we have reproduced the Brown-Henneaux asymptotic Virasoro algebra with the central charge $6 k$.

\section{General solution of the Chern-Simons theory and Relation to the Wess-Zumino-Witten model}

In this section we derive the general solution of the Chern-Simons theory (2.9) and show its relation to the boundary theory. Let us solve the equations of motion $F_{A}=F_{B}=0$ under the appropriate boundary condition (2.5). Thanks to the field equations, the gauge fields become pure gauge form

$$
A_{m}=g^{-1} \partial_{m} g, \quad B_{m}=-\partial_{m} \bar{g} \bar{g}^{-1}
$$

Constrained by the boundary condition (2.5), the group elements $g(t, \phi, \rho)$ and $\bar{g}(t, \phi, \rho)$ become functions of $t+\phi$ and $t-\phi$ only on the boundary, respectively; $h(t+\phi)$ and $\bar{h}(t-\phi)$. Such group elements define the space of connection $\mathcal{A}$. Next, we would like to divide the space of connections $\mathcal{A}$ by the space of gauge functions. Let us define the space of gauge function $\hat{G}_{0}$ such that any element in $\hat{G}_{0}$ becomes the identity on the boundary. Now, the space of gauge inequivalent classes is $\mathcal{A} / \hat{G}_{0}$. If we restrict these spaces $\mathcal{A}$ and $\hat{G}_{0}$ inside the boundary, these spaces become exactly the same. Then, the quotient space $\mathcal{A} / \hat{G}_{0}$ will be expected as the space of the boundary degrees of freedom $h(t+\phi)$ and $\bar{h}(t-\phi)$. Let us show this fact. The gauge degrees of freedom $\hat{G}_{0}$ are equally fixed by imposing the gauge fixing condition

$$
A_{\rho}=\beta+\alpha, \quad B_{\rho}=\beta-\alpha,
$$

where $\alpha$ and $\beta$ are any constant element of $S L(2 ; \mathbf{R})$. This follows from the fact that the residual gauge transformation

$$
\begin{aligned}
& A_{\rho}=f^{-1} A_{\rho} f+f^{-1} \partial_{\rho} f, \\
& B_{\rho}=\bar{f}^{-1} B_{\rho} \bar{f}+\bar{f}^{-1} \partial_{\rho} \bar{f},
\end{aligned}
$$


is easily integrated giving the elements of $\hat{G}_{0}$ as $f=\bar{f}=1$. After imposing the gauge fixing condition, the group elements $g$ and $\bar{g}$ take a form $g=h(t, \phi) e^{(\alpha+\beta) \rho}$ and $\bar{g}=e^{(\alpha-\beta) \rho} \bar{h}(t, \phi)$. Now, imposing the boundary condition (2.5), we finally obtain

$$
g=h(t+\phi) e^{(\alpha+\beta) \rho}, \quad \bar{g}=e^{(\alpha-\beta) \rho} \bar{h}(t-\phi) .
$$

At first sight one might think that since the solution (5.1) took a pure gauge form, the solution would be gauged away completely. However, our result (5.4) illustrates that the boundary degrees of freedom $h(t+\phi)$ and $\bar{h}(t-\phi)$ survive as the physical degrees of freedom of the system. These group elements with eqs. (5.1) give quite general solutions of the Chern-Simons gravity. Since the metric should not degenerate, $\alpha \neq 0$ is assumed. In this situation, the $g_{\rho \rho}$ part of the metric can be set equal to unity $g_{\rho \rho}=1$ by rescaling the radial variable $\rho$, and then we may put $\alpha_{a} \alpha^{a}=1$. For later simplicity we set $\alpha=J_{2}$ and $\beta=0$.

According to the solution (5.4), the dreibein and spin-connection become

$$
\begin{array}{lll}
e_{+}=\frac{1}{2} e^{-\alpha \rho} h^{-1} \partial_{+} h e^{\alpha \rho}, & \omega_{+}=e_{+}, \\
e_{-}=\frac{1}{2} e^{\alpha \rho} \partial_{-} \bar{h} \bar{h}^{-1} e^{-\alpha \rho}, & \omega_{-}=-e_{-}, \\
e_{\rho}=\alpha, & \omega_{\rho}=0,
\end{array}
$$

where the subindices \pm indicate that quantities $f_{ \pm}$are the $d t \pm d \phi$ components of oneforms $f=e^{a}, \omega^{a}, d$. This is the most general gauge-fixed solution of the system (2.9). The black hole metric becomes

$$
\begin{aligned}
g_{++} & =\frac{1}{2} \operatorname{tr}\left(h^{-1} \partial_{+} h\right)^{2} \\
g_{--} & =\frac{1}{2} \operatorname{tr}\left(\partial_{-} \bar{h} \bar{h}^{-1}\right)^{2} \\
g_{+-} & =\frac{1}{2} \operatorname{tr}\left(e^{-2 \alpha \rho} h^{-1} \partial_{+} h e^{2 \alpha \rho} \partial_{-} \bar{h} \bar{h}^{-1}\right) \\
g_{\rho+} & =\operatorname{tr}\left(\alpha h^{-1} \partial_{+} h\right) \\
g_{\rho-} & =\operatorname{tr}\left(\alpha \partial_{-} \bar{h} \bar{h}^{-1}\right) \\
g_{\rho \rho} & =1
\end{aligned}
$$

where the trace is taken on the subgroup $H$. At infinity $\rho \sim \infty$, the asymptotic behavior of the metric is

$$
g_{+-} \sim \mathcal{O}\left(e^{2 \rho}\right)
$$

and the others are of $\mathcal{O}(1)$. This behavior provides us with the same isometry solution (3.12) again. The solution (5.6) shows that the metric is specified by an infinite number 
of conserved currents, i.e., the Kac-Moody currents. We note that the BTZ black hole 17 corresponds to the special choice

$$
h=e^{z_{+} J_{1} \cdot(t+\phi)}, \quad \bar{h}=e^{z_{-} J_{1} \cdot(t-\phi)} .
$$

And the vacuum solution corresponding to the anti-de-Sitter space is not represented by (5.8) for any $z_{ \pm} \in \mathbf{R}$ but is given by

$$
h=e^{J_{0} \cdot(t+\phi)}, \quad \bar{h}=e^{J_{0} \cdot(t-\phi)},
$$

by which we find

$$
\begin{aligned}
e & =J_{0} \cosh \rho d t+J_{1} \sinh \rho d \phi+J_{2} d \rho, \\
w & =J_{0} \cosh \rho d \phi+J_{1} \sinh \rho d t .
\end{aligned}
$$

The two dimensional parts $h(t+\phi)$ and $\bar{h}(t-\phi)$ of the solution (5.4) also can be given by the Wess-Zumino-Witten action

$$
I_{ \pm}(h)=-\frac{k}{4 \pi} \operatorname{tr} \oint \partial_{+} h^{-1} \partial_{-} h \mp \frac{k}{12 \pi} \operatorname{tr} \int\left(h^{-1} d h\right)^{3}
$$

and, then, the action of the boundary theory would become $L=I_{+}(h)+I_{-}(\bar{h})$. Unfortunately, the symmetry of this action is twice as large as that of the boundary theory. We have to throw away the modes represented by $-\partial_{-} h h^{-1}$ and $\bar{h}^{-1} \partial_{+} \bar{h}$. Instead of this choice, we have more excellent one. Suppose that the representations of the left- and right-handed generators are the same. Let us consider the Wess-Zumino-Witten theory $I_{+}(g)$ with the subgroup gauge symmetry $H$. Then, the field equation $\partial_{-}\left(g^{-1} \partial_{+} g\right)=0$ provides us with the two conserved currents $g^{-1} \partial_{+} g=h^{-1} \partial_{+} h$ and $-\partial_{-} g g^{-1}=-\partial_{-} \bar{h}^{-1}$, by regarding the identification $g=\bar{h}(t-\phi) h(t+\phi)$. Then, in this situation the action of the boundary theory becomes

$$
L=I_{+}(g)
$$

Finally, let us show that after imposing the constraints the boundary term in $\delta L$, namely

$$
0=\frac{k}{4 \pi} \operatorname{tr} \oint\left(\delta A_{+} A_{-}+\delta B_{-} B_{+}\right)
$$

becomes the field equation of the boundary theory (5.12).

The constraint is given by the variation of $L$ with respect to the multipliers $A_{t}$ and $B_{t}$, so we have $F_{A \rho \phi}=F_{B} \rho \phi=0$. These are easily integrated to be $A_{\phi}=e^{-\alpha \rho} J(t, \phi) e^{\alpha \rho}$ and $B_{\phi}=e^{\alpha \rho} \bar{J}(t, \phi) e^{-\alpha \rho}$. Since the quantity $J(t, \phi)(\bar{J}(t, \phi))$ should be a $\phi$ component of 
a one-form and be Lie algebra valued, they should be given by the Maurer-Cartan oneforms $J=h^{-1} \partial_{\phi} h$ and $\bar{J}=-\partial_{\phi} \bar{h} \bar{h}^{-1}$ for some appropriate group elements $h(t, \phi)$ and $\bar{h}(t, \phi)$. Since $A_{\rho}$ and $B_{\rho}$ are gauge-fixed, we have to impose the Gauss law constraints $\delta L / \delta A_{\rho}=\delta L / \delta B_{\rho}=0$ which lead to the conditions

$$
\begin{aligned}
\partial_{t} J & =\partial_{\phi} K+[J, K], \\
\partial_{t} \bar{J} & =\partial_{\phi} \bar{K}+[\bar{J}, \bar{K}],
\end{aligned}
$$

where we put $K=e^{-\alpha \rho} A_{t} e^{\alpha \rho}$ and $\bar{K}=e^{\alpha \rho} B_{t} e^{-\alpha \rho}$. These determine the multipliers $A_{t}$

and $B_{t}$ as $K=h^{-1} \partial_{t} h$ and $\bar{K}=-\partial_{t} \bar{h} \bar{h}^{-1}$. We note that the variations with respect to $\delta A_{\phi}$ and $\delta B_{\phi}$ are the field equation, which becomes identity equation after imposing the constraints. Now, using the above constraints, the boundary condition from the boundary term is equivalent to

$$
0=\frac{k}{4 \pi} \operatorname{tr} \oint\left[h^{-1} \delta h \partial_{-}\left(h^{-1} \partial_{+} h\right)+\delta \bar{h} \bar{h}^{-1} \partial_{+}\left(\partial_{-} \bar{h} \bar{h}^{-1}\right)\right] .
$$

This is nothing but the variational equation of the Wess-Zumino-Witten action (5.12); $\delta I_{+}(g)=0$ with the identification of the solution $g(t, \phi)=\bar{h}(t-\phi) h(t+\phi)$. In other words, after imposing the constraints, the boundary condition of the bulk theory becomes the field equation of the boundary theory.

In conclusion, the boundary CFT theory of the $A d S_{3}$ gravity theory (2.9) is equivalent to the Wess-Zumino-Witten theory (5.12). The usual energy-momentum tensor takes the Sugawara form as is easily derived by the Noether method. However, this is not exactly the generator of the asymptotic symmetry, but merely a part of it. The correct generator is the energy-momentum tensor (4.12) twisted by the contribution of the local rotation by the vector-like gauge subgroup $S L(2 ; \mathbf{R})_{L+R}$.

\section{Quantization of Chern-Simons theory}

When the system is quantized, the Poisson's bracket is replaced by the commutator bracket; $i\{,\}_{P} \rightarrow[$,$] . Immediately, we obtain the two Kac-Moody algebras of cen-$ ter $k$. In quantized version, the coefficient of the linear term is modified in the twisted energy-momentum tensor

$$
\begin{aligned}
& T \rightarrow T+\frac{k}{k-2} \partial_{+} J^{2} \\
& \bar{T} \rightarrow \bar{T}+\frac{k}{k-2} \partial_{-} \bar{J}^{2}
\end{aligned}
$$


As usual, the currents in the definition of the Virasoro generators (4.12) should be normal ordered like

$$
: J_{n}^{a} J_{m}^{b}:=\left\{\begin{array}{ll}
J_{n}^{a} J_{m}^{b} & (n \leq m) \\
J_{m}^{b} J_{n}^{a} & (m<n)
\end{array},\right.
$$

and similarly for $\bar{J}_{n}^{a}$. Then, the definition of the Virasoro generators are modified to be

$$
\begin{aligned}
& L_{n}=\frac{1}{k-2} \sum_{m}: J_{a m} J_{n-m}^{a}:+\frac{k}{k-2} i n \alpha_{a} J_{n}^{a}+a_{0} \delta_{n}, \\
& \bar{L}_{n}=\frac{1}{k-2} \sum_{m}: \bar{J}_{a m} \bar{J}_{n-m}^{a}:-\frac{k}{k-2} i n \alpha_{a} \bar{J}_{n}^{a}+a_{0} \delta_{n},
\end{aligned}
$$

where we added a constant $a_{0}=\frac{k^{3}}{4(k-2)^{2}}$. The commutation relations are

$$
\begin{aligned}
& {\left[L_{n}, L_{m}\right]=(n-m) L_{n+m}+\frac{c}{12} n\left(n^{2}-1\right) \delta_{n+m},} \\
& {\left[\bar{L}_{n}, \bar{L}_{m}\right]=(n-m) \bar{L}_{n+m}+\frac{c}{12} n\left(n^{2}-1\right) \delta_{n+m},}
\end{aligned}
$$

where

$$
c=\frac{3 k}{k-2}+6 k\left(\frac{k}{k-2}\right)^{2} .
$$

At weak coupling limit $k \rightarrow \infty$ the center (6.5) takes the classical value $6 k$ in the leading order.

The physical state condition is

$$
\begin{array}{ll}
\left.\left.J_{0}^{a} \mid \text { phys }\right\rangle=\mid \text { phys }\right\rangle J^{a}, & \left.\left.L_{0} \mid \text { phys }\right\rangle=h_{0} \mid \text { phys }\right\rangle, \\
\left.J_{n}^{a} \mid \text { phys }\right\rangle=0, & \left.L_{n} \mid \text { phys }\right\rangle=0,
\end{array}
$$

for $n=1,2,3, \cdots$, where $h_{0}=J_{a} J^{a} /(k-2)+a_{0}$.

\section{Summary and discussions}

We have analyzed the Chern-Simons gravity theory with the boundary term (2.8). We introduced the boundary term $\mathcal{B}$ in the action $I_{C S}(B)-I_{C S}(A)$ such that the condition $\delta \mathcal{B}+\delta \mathcal{B}^{\prime}=0$ uniquely provides us with the boundary condition (2.5). If we did not introduce the boundary term, the boundary theory would be a pair of the chiral WessZumino-Witten theory

$$
I_{c \pm}(g)=-\frac{k}{4 \pi} \operatorname{tr} \oint \partial_{t} g^{-1} \partial_{\phi} g \mp \frac{k}{12 \pi} \operatorname{tr} \int\left(g^{-1} d g\right)^{3},
$$

in which theory the Kac-Moody currents would not be guaranteed to be a function only of $t \pm \phi$. This theory does not describe the asymptotic behavior of the Chern-Simons theory 
with the boundary condition (2.5). In other words, without the boundary term $\mathcal{B}$, the gauge functions $u$ and $v$ for the asymptotic symmetry (4.10) would not be guaranteed to consist of the left- and right-moving modes, respectively, since $A_{\phi}$ and $B_{\phi}$ is not a function of $t+\phi$ and $t-\phi$, respectively. This result mismatches the fact that the asymptotic symmetry (4.10) is a function of $t+\phi$ or $t-\phi$.

The Brown-Henneaux asymptotic symmetry should be exclusively derived from the condition (3.7). The most important point is that the diffeomorphism should be accompanied by the manifest gauge transformation in $H$ through eqs. (3.1) in the Chern-Simons formulation. In our formulation the gauge functions $u$ and $v$ for the asymptotic symmetry (4.10) are naturally made of the left- and right-moving modes, respectively. Unless we consider the gauge function $w^{a}$ in (3.1), one would be forced to introduce two kinds of diffeomorphism parameter $\xi^{m}$ in $u^{a}$ and $v^{a}$ separately. 13, 19 This is unfavorable.

We have found the general solution (5.4) of the $A d S_{3}$ Chern-Simons gravity. Dividing

the space of solutions $\mathcal{A}$ by the space of gauge transformations $\hat{G}_{0}$, the resultant general solution is characterized by $h(t+\phi)$ and $\bar{h}(t-\phi)$ which are boundary degrees of freedom of the bulk theory.

The physical boundary degrees of freedom $h(t+\phi)$ and $\bar{h}(t-\phi)$ of the $A d S_{3}$ gravity theory (2.9) are described by the Wess-Zumino-Witten theory (5.12). Then, we have written down the quite general form of the metric (5.6) in terms of the Kac-Moody currents. The BTZ black hole is included in our black hole solutions.

The classical central charge of the Virasoro algebra is $6 k$, while the quantum counterpart becomes eq. (6.5).

\section{Acknowledgements}

The work of T.K. was supported in part by Scientific Grants from the Ministry of Education (Grant Number, 09640353).

\section{References}

[1] J. Maldacena, Adv. Theor. Math. Phys. 2 (1998) 231, hep-th/9711200,

"THE LARGE N LIMIT OF SUPERCONFORMAL FIELD THEORIES AND SUPERGRAVITY". 
[2] S.S. Gubser, I.R. Klebanov and A.W. Peet, Phys. Rev. D54, (1996) 3915, hepth/9602135,

"Entropy And Temperature Of Black 3-Branes".

[3] I.R. Klebanov, Nucl. Phys. B496, (1997) 231, hep-th/9702076,

"World Volume Approach To Absorption By Nondilatonic Branes".

[4] S.S. Gubser, I.R. Klebanov and A.A. Tseytlin, Nucl. Phys. B499, (1997) 217, hepth/9703040,

"String Theory And Classical Absorption By Three-branes".

[5] S.S. Gubser and I.R. Klebanov, Phys. Lett. B413, (1997) 41, hep-th/9708005,

"Absorption By Branes And Schwinger Terms In The World Volume Theory".

[6] J. Maldacena and A. Strominger, JHEP 19, (1997) 9712, hep-th/9710014,

"Semiclassical Decay Of Near Extremal Fivebranes".

[7] S.S. Gubser and I.R. Klebanov and A.M. Polyakov, Phys. Lett. B428, (1998) 105, hep-th/9802109,

"Gauge Theory Correlators From Noncritical String Theory".

[8] E. Witten, Adv. Theor. Math. Phys. 2 (1998) 253, hep-th/9802150,

"ANTI DE SITTER SPACE AND HOLOGRAPHY".

[9] J.D. Brown and M. Henneaux, Commun. Math. Phys. 104 (1986) 207,

"Central Charges in the Canonical Realization of Asymptotic Symmetries: An Example from Three Dimensional Gravity".

[10] T. Regge and C. Teitelboim, Ann. Phys. 88 (1974) 286,

"Role of surface integrals in the Hamiltonian formulation of general relativity".

[11] A. Achúcarro and P.K. Townsend, Phys. Lett. 180B (1986) 89,

"A CHERN-SIMONS ACTION FOR THREE-DIMENSIONAL ANTI-DE-SITTER SUPERGRAVITY THEORIES".

[12] E. Witten, Nucl. Phys. B311 (1988/89) 46, "2+1 DIMENSIONAL GRAVITY AS AN EXACTLY SOLUBLE SYSTEM".

[13] M. Bañados, Phys. Rev D52 (1996) 5816, hep-th/9405171,

"Global charges in Chern-Simons theory and the $2+1$ black hole"; 
M. Bañados, hep-th/9901148,

"Three-dimensional quantum geometry and black holes".

[14] S. Carlip, Phys. Rev. D51 (1995) 632,

"Statistical mechanics of the (2+1)-dimensional black hole".

[15] K. Ezawa, Int. J. Mod. Phys. A10 (1995) 4139,

" "Moduli space" of asymptotically anti-de-Sitter space-times in $2+1$ dimensions".

[16] P. Oh and M.-I. Park, Mod. Phys. Lett. A14 (1999) 231, hep-th/9805178,

"SYMPLECTIC REDUCTION AND SYMMETRY ALGEBRA IN BOUNDARY CHERN-SIMONS THEORY";

M.-I. Park, Nucl. Phys. B544 (1999) 377, hep-th/9811033,

"Symmetry Algebras in Chern-Simons Theories with Boundary: Canonical Approach".

[17] M. Bañados, C. Teitelboim and J. Zanelli, Phys. Rev. Lett. 69 (1992) 1849, "Black Hole in Three-Dimensional Spacetime".

[18] O. Coussaert, M. Henneaux and P. van Driel, Class. Quant. Grav. 12 (1995) 2961, gr-qc/9506019,

"The asymptotic dynamics of three-dimensional Einstein gravity with a negative cosmological constant".

[19] M. Bañados, T. Brotz and M.E. Ortiz, hep-th/9802076,

"Boundary dynamics and the statistical mechanics of the $2+1$ dimensional black hole".

[20] K. Behrndt, I. Brunner and I. Gaida, Phys. Lett. 180B (1986) 89, hep-th/9806195, "AdS $S_{3}$ Gravity and Conformal Field Theories".

[21] M. Bañados, M. Henneaux, C. Teitelboim and J. Zanelli, Phys. Rev D48 (1993) 1506, "Geometry of the $2+1$ black hole". 